\title{
Hadronic versus leptonic origin of the gamma-ray emission from supernova remnant RX J1713.7-3946
}

\author{
E. G. Berezhko ${ }^{1}$ and H. J. Völk ${ }^{2}$
}

\author{
1 Yu.G. Shafer Institute of Cosmophysical Research and Aeronomy, 31 Lenin Ave., 677980 Yakutsk, Russia \\ e-mail: berezhko@ikfia.ysn.ru \\ 2 Max Planck Institut für Kernphysik, Postfach 103980, 69029 Heidelberg, Germany \\ e-mail: Heinrich.Voelk@mpi-hd.mpg.de
}

Received 23 June 2008 / Accepted 25 August 2008

\begin{abstract}
Aims. The hadronic vs. leptonic origin of the gamma-ray emission from the Supernova Remnant RX J1713.7-3946 is discussed both in the light of new observations and from a theoretical point of view.

Methods. The existing good spatial correlation of the gamma-ray emission and the nonthermal X-ray emission is analyzed theoretically. In addition, the recently published new HESS observations define the energy spectrum more precisely, in particular at the high and low energy ends of the instrument's dynamical range. There now exist much more constraining X-ray observations from Suzaku that extend substantially beyond $10 \mathrm{keV}$. These new data are compared with the authors' previous theoretical predictions, both for dominant hadronic and for simple inverse Compton models.

Results. Apart from the well-known MHD correlation between magnetic field strength and plasma density variations, emphasized by the wind-bubble-structure of the remnant, it is argued that the regions of magnetic field amplification also are correlated with enhanced densities of accelerated nuclear particles and the associated streaming instabilities. Therefore a correlation of nonthermal X-ray and $\gamma$-ray emission is not only possible but even to be expected for a hadronic emission scenario. A leptonic origin of the gamma-ray emission would require an implausibly uniform strength of the magnetic field. The observational and theoretical inferences about substantial field amplification in this remnant agree very well with the recent X-ray and $\gamma$-ray observations.

Conclusions. All this argues strongly for the dominance of hadronic $\gamma$-rays in the $\gamma$-ray emission spectrum and a fortiori for an overwhelming contribution of nuclear cosmic ray particles to the nonthermal energy in this remnant.
\end{abstract}

Key words. ISM: cosmic rays - acceleration of particles - shock waves - stars: supernovae: individual: SNR RX J1713.7-3946 radiation mechanisms: non-thermal - gamma rays: theory

\section{Introduction}

Hard X-ray emission from young Supernova Remnants (SNRs) was first detected in SN 1006 and interpreted as synchrotron emission from electrons in the energy range of tens of $\mathrm{TeV}$ (Koyama et al. 1995). Such emission meanwhile has been found in many young shell-type objects. These electrons are also expected to produce very high energy (VHE) $\gamma$-rays in the $>100 \mathrm{GeV}$ range by inverse Compton (IC) collisions with low energy photons from the ambient radiation field, in particular the Cosmic Microwave Background (CMB). In regions of high gas density this may also be accompanied by a nonnegligible emission of nonthermal Bremsstrahlung $\gamma$-rays. It is therefore not a priori clear whether the VHE $\gamma$-rays detected in several shell-type SNRs up to now - Cas A (Aharonian et al. 2001a; Albert et al. 2007), RX J1713.7-3946 (Muraishi et al. 2000; Enomoto et al. 2002; Aharonian et al. 2004, 2006, 2007a), RX J0852.0-4622 (Katagiri et al. 2005; Aharonian et al. 2005, 2007b; Enomoto et al. 2006), and RCW 86 (Hoppe et al. 2007) - are indeed the result of inelastic collisions of energetic nuclei with gas atoms, as expected theoretically if a sizeable fraction of the SN explosion energy goes into nuclear cosmic rays (CRs) (Drury et al. 1994; Berezhko \& Völk 1997), or whether they are merely due to IC collisions of the X-ray synchrotron emitting electrons.
The latter inference seems at least in a rough sense possible if the effective magnetic field strength in these objects is sufficiently low. Then the energy spectrum of accelerated electrons, calculated from the observed synchrotron spectrum, contains sufficiently many particles so that a strong enough IC $\gamma$-ray emission results. Since magnetic fields are difficult to determine, a possible phenomenological approach is to assume that the interior (postshock) magnetic field in the SNR is the result of MHDcompression of a circumstellar field whose strength is at best a few $\mu \mathrm{G}$. For RX J1713.7-3946 and RX J0852.0-4622 such a procedure works, at least as far as the overall amplitude of the $\gamma$-ray spectrum is concerned. For Cas A this is true as well. However, all these remnants presumably correspond to core collapse SN explosions into the wind bubble of a massive progenitor star, with a rather complex circumstellar magnetic field structure. In contrast, for the remnants of several type Ia SNe which could not be detected so far in $\gamma$-rays such as Tycho's SNR, SN 1006, and Kepler's SNR, this scheme leads to large overpredictions for the $\gamma$-ray flux, unless the mean interior magnetic field strength $B$ is assumed to be significantly higher than the value $\sim 10 \mu \mathrm{G}$ expected from a $\lesssim 5 \mu \mathrm{G}$ circumstellar field for a strong quasi-parallel, adiabatic shock with a compression ratio of 4 (Völk et al. 2008a, b). Since electron acceleration cannot be responsible for such an amplification of the interstellar field, this 
makes a pure electron acceleration scenario generally untenable in our view.

The acceleration of nuclear particles on the other hand cannot be deduced in a similar way from some other electromagnetic emission - even though the magnetic field strength and the ion injection rate are inferred from the form of the electron synchrotron spectrum (Berezhko et al. 2002). The energetic nuclear component needs to be calculated theoretically, and the amplitude of the accelerated particle distribution can only result from a nonlinear theory. This has been done successfully in a number of cases and shows that the overall energy in accelerated nuclei is indeed a large fraction - about $10 \%$ - of the available hydrodynamic explosion energy, and that the local efficiency at those parts of the shock surface, where ion injection is effective, amounts to even about 50\% (see e.g. Berezhko 2005, for a review).

Given this high acceleration efficiency, at reasonable thermal gas densities the expected rate of hadronic collisions with gas atoms and the resulting $\gamma$-ray production by $\pi^{0}$-decay turns out to be consistent with the observations in the $\gamma$-ray detected objects analyzed so far: Cas A (Berezhko et al. 2003b), RX J1713.7-3946 (Berezhko \& Völk 2006, hereafter referred to as BV06), (Berezhko \& Völk 2007), RX J0852.0-4622 (Berezhko et al. 2008). Also for the other objects that we have analyzed theoretically - Tycho's SNR (Völk et al. 2002, 2005, 2008), SN 1006 (Berezhko et al. 2002; Ksenofontov et al. 2005), and Kepler's SNR (Berezhko et al. 2006) - and for which only upper limits exist so far, the hadronic $\gamma$-ray emission is expected to dominate the IC fraction, even though for the low-density object SN 1006 only by a modest margin.

Within the errors, the theoretically derived magnetic field strengths also agree with those deduced from the observations of filamentary X-ray synchrotron features, often found at the outer rims of the SNRs (e.g. Vink \& Laming 2003; Long et al. 2003; Bamba et al. 2003; Berezhko et al. 2003a; Berezhko \& Völk 2004; Völk et al. 2005; Parizot et al. 2006). The field strengths typically are an order of magnitude greater than the compressed circumstellar field, substantially reducing the IC spectrum amplitude for a given synchrotron spectrum and thus tending to yield a negligible leptonic contribution to the observed VHE $\gamma$-ray flux.

Although the dominant acceleration of nuclear particles is clearly favored by what has been said up to now, leptonic scenarios have been studied in some detail (e.g. Porter et al. 2006; Katz \& Waxman 2008). Especially for the experimentally beststudied SNR RX J1713.7-3946 the question is whether an IC spectrum, scaled in amplitude from the synchrotron spectrum in the sense described above, is compatible with the form of the observed $\gamma$-ray spectrum. A second question regards the observed spatial correlations of the morphology in hard X-rays, assumed to be the result of synchrotron radiation, and in $\gamma$-rays. Such a correlation has been observed in RX J1713.7-3946 and might at first sight be attributed to a common leptonic population of energetic particles. It remains to be seen whether such an inference holds upon deeper scrutiny.

Another, not quite resolved question concerns the absence of thermal X-ray emission in the two SNRs RX J1713.7-3946 and RX J0852.0-4622 that are spatially resolved at TeV energies. Also the radio synchrotron emission is very weak in comparison to the strong X-ray synchrotron emission in both sources. Since the earliest observations and analyses (Slane et al. 1999), the explanation for this situation has been the assumption that the $\mathrm{SN}$ explosion occurred into the very rarefied stellar wind bubble of a massive progenitor star. The model of BV06 specifies the bubble structure in detail. It demonstrates that the main gas heating and particle acceleration occurs beyond the wind region and bubble, when the SNR shock propagates into the swept-up, radiatively cooled shell of interstellar matter that was generated by the forward shock driven into the ambient ISM by the expansion of the wind bubble. Therefore the wind bubble has two main effects: the SNR can reach a large size quickly and it is then rather quickly decelerated in the dense shell. During propagation of the SNR shock wave through the wind and bubble the gas density is very low, resulting in a very low thermal X-ray emission of this material. The shock's late encounter of the massive shell heats the nuclear particles, but leaves little time for the subsequent relaxation in the postshock region of the SNR that heats the thermal electrons by Coulomb collisions with the heavy ions. In this way a minimum of thermal X-ray emission from the remnant is combined with a maximum of hard X-ray synchrotron and hadronic $\gamma$-ray emission from the SNR. In addition, the shock is nonlinearly modified. For a SNR propagating into a uniform medium with a uniform magnetic field this implies that the larger part of the shock surface corresponds to a quasi-perpendicular shock with a strongly reduced injection of nuclear particles (Völk et al. 2003). Suprathermal injection of ions is only possible in the quasi-parallel shock regions. If the spatial scales of the quasi-perpendicular regions are large enough, the cross-field diffusion of the highest-energy particles from the neighboring quasi-parallel shock regions also does not reach deeply into these quasi-perpendicular regions. In the corresponding magnetic flux tubes no magnetic field amplification occurs either and the shock remains essentially un-modified there. This means that in the quasi-perpendicular regions the shock dissipation and therefore the gas heating occurs in a locally unmodified shock with the overall shock speed, leading to a rather high gas temperature. In the case where a radiatively cooling shell of a wind bubble is the main obstacle for the SNR expansion, the situation may be different. The MHD instabilities of such a shell probably break it into many small regions with strongly varying field directions. Then the spatial scales separating the quasi-perpendicular from the quasi-parallel shock regions may become small enough that crossfield diffusion can smear out the quasi-perpendicular regions and particle acceleration occurs practically everywhere over the shock surface (Völk et al. 2007; Völk 2008). In the extreme this implies shock modification over the entire shock region and thus a reduced gas heating due to the subshock dissipation only ${ }^{1}$. Compared to an un-modified shock of the same overall speed, the gas temperature is then diminished by a factor $\approx \sigma_{\mathrm{s}}^{2} / \sigma^{2} \approx 0.25$, where $\sigma$ and $\sigma_{\mathrm{s}}$ denote the overall shock compression ratio and the subshock compression ratio, respectively. This reduces the emission of soft thermal X-rays drastically.

Such a configuration is not easily analysed in detail with standard methods of X-ray astronomy. However, this also leaves uncertainties in the evaluation of the model's validity. Here, as in BV06, we proceed under the assumption that the concrete wind bubble model, or its eventual improvement, is consistent with the fact that no thermal emission has been found up to now.

The purpose of the present paper is a discussion of the above two questions for RX J1713.7-3946, even though we expect analogous arguments to hold for the other objects mentioned. In Sect. 2 we discuss the correlations between the gas density and the magnetic field strength that are possible, or are even to be expected, in the circumstellar medium before the SN explosion. In

\footnotetext{
1 The given $\gamma$-ray flux requires in addition a reduction of the gas density which reduces the thermal emission.
} 
an approximate way we also show how variations in gas density lead to correlated variations in nuclear CR pressure and in the hadronic $\gamma$-ray emission. However, we shall argue that the magnetic field direction influences particle injection into the shock acceleration process, so that circumstellar density enhancements are only a necessary but not a sufficient condition for enhancements of the $\gamma$-ray emission. Under most circumstances electron synchrotron emission is then expected to increase with hadronic $\gamma$-ray emission as well. We also briefly discuss the alternative purely leptonic scenario. In Sect. 3 we compare our calculations for the hadronic $\gamma$-ray emission, the synchrotron emission, and for the IC and Bremsstrahlung $\gamma$-ray emission with the latest $\mathrm{X}$-ray and $\gamma$-ray measurements for RX J1713.7-3946. The results are finally compared with a purely leptonic scenario, calculated by assuming ion injection and thus also magnetic field amplification to be negligibly small. It turns out that a hadronic origin of the $\gamma$-ray emission is consistent with all measurements and with our theory, whereas the leptonic scenario runs into serious difficulties. Section 4 contains our conclusions.

Very recently an independent discussion of these questions by Tanaka et al. (2008) has come to our attention. Their paper is more observation oriented and is partly complementary to ours. It uses phenomenological estimates for the accelerated particle spectra. However their conclusions are similar to ours.

\section{Expected morphological correlations}

In this section we investigate the spatial correlations that arise from compressions or expansions of the thermal gas with a frozen-in magnetic field, in a given radiation field. In addition we argue that variations of the nuclear energetic particle density - which are most likely the result of spatial variations of the injection rate into the acceleration process - lead to positively correlated enhancements of the magnetic field strength as a result of magnetic field amplification by CR streaming instabilities.

\subsection{Compressions/de-compressions of the thermal plasma}

Consider briefly quasi-static compressions/de-compressions of the gas density by plasma motions across the magnetic field in the MHD limit.

In this case the conservation of magnetic flux compresses the field together with the thermal gas, increasing at the same time the target for electrons to produce synchrotron emission as well as the target for energetic nuclei in inelastic collisions with the gas atoms. In both cases the densities of the X-ray synchrotron emitting electrons and of the $\gamma$-ray emitting nuclei vary approximately in the same way, because the electrons and nuclei concerned have roughly the same energy. Therefore their spatial distributions are essentially the same.

In the 1-fluid MHD-approximation the mass density $\rho$ and the magnetic field vector $\boldsymbol{B}$ are related to the mass velocity $\boldsymbol{u}$ through the conditions of conservation of mass and magnetic flux:

$\frac{\partial \rho}{\partial t}+\boldsymbol{u} \cdot \nabla \rho \equiv \frac{\mathrm{d} \rho}{\mathrm{d} t}=-\rho \nabla \cdot \boldsymbol{u}$

$\frac{\partial \boldsymbol{B}}{\partial t}=\nabla \times(\boldsymbol{u} \times \boldsymbol{B})$
Being interested primarily in the variations of $\rho$ and the magnitude $B$ of $\boldsymbol{B}$, it is more useful for our considerations to reduce Eqs. (1-2) to equations for $B^{2}$ and $B / \rho$ alone, using $\nabla \cdot \boldsymbol{B}=0$ :

$$
\begin{aligned}
& \frac{1}{2 B^{2}} \frac{\mathrm{d} B^{2}}{\mathrm{~d} t}=-\nabla \cdot \boldsymbol{u}-\boldsymbol{B} \cdot(\boldsymbol{B} \cdot \nabla) \boldsymbol{u} \\
& \frac{\mathrm{d} \ln (B / \rho)}{\mathrm{d} t}=B^{-2} \boldsymbol{B} \cdot(\boldsymbol{B} \cdot \nabla) \boldsymbol{u},
\end{aligned}
$$

where the r.h.s. of Eq. (3) is nonzero only for an expansion/compression perpendicular to the direction of $\boldsymbol{B}$ and Eq. (4) shows that the ratio $B / \rho$ changes only in an expansion/compression parallel to $\boldsymbol{B}$.

With these relations in mind we consider the circumstellar medium of a single massive star before it is reached by the SNR shock. The stellar wind produces an expanding hot, low density bubble. This dynamical evolution compresses the ambient interstellar medium (ISM) in an outer shock wave in an approximately spherically symmetric manner. It is clear that the compression of this external medium will create a shocked shell with the gas density increasing radially outwards and, over most of the solid angle (where the interstellar B-field is not radial), also an increasing magnetic field strength. All pre-existing density inhomogeneities in the external medium in the form of clouds will by themselves have generally higher B-fields, wherever the density is high. The subsequent compression by the wind bubble will tend to enhance these correlated density and B-field contrasts. Azimuthal density variations will probably form upon this compression as well, by azimuthal instabilities of the radiatively cooling swept-up shell, regardless of pre-existing clouds. All this implies spatially correlated variations of $\rho$ and $B$ in the medium upstream of the SNR shock.

As the theoretical model of BV06 suggests, the remnant of the subsequent $\mathrm{SN}$ explosion should be old enough that the swept-up mass of interstellar matter dominates the mass enclosed in the wind bubble as well as the ejected mass from the explosion. Then we have the situation that most of the emitting medium is a shock-modified ISM that originally had a structure as described above. Its nonthermal emission dominates the $\gamma$-ray emission as well as the synchrotron emission of the SNR, as we observe it today.

\subsection{Injection and acceleration of nuclear particles at the SNR shock}

If the magnetic field lines in front of the accelerating forward SNR blast wave are quasi-perpendicular to the shock normal, suprathermal ions cannot escape from the downstream region (i.e. from the remnant interior) into the upstream region to start being diffusively accelerated. On the other hand, nuclear particles can be readily injected in quasi-parallel shock regions where the field lines are more or less parallel to the shock normal. And the number of ions injected into the acceleration from each gas volume is to a first approximation proportional to the thermal gas density. In such field regions a high energy density of accelerated nuclear particles will be built up by the acceleration process. This particle population will try to escape into the upstream medium and thereby excite streaming instabilities which lead to magnetic field amplification in the associated magnetic flux tubes. The enhanced field amounts at the same time to an increased target strength for synchrotron emission by electrons, while the enhanced energetic nuclear particle intensity will increase the number of inelastic collisions per unit volume at given 
gas density. As already mentioned in the Introduction an inhibition of acceleration in quasi-perpendicular shock regions may not occur in the majority of quasi-perpendicular regions of the shock because of cross-field diffusion. However, in principle this effect is there and may occur especially at pre-existing interstellar clouds encountered by the SNR shock.

The injection of energetic electrons into the acceleration process is not very well understood, and is possibly not correlated with the ion injection. Electron injection might actually occur everywhere over the SNR shock surface. It may or may not increase with the plasma density.

Let us for a moment assume that electron injection increases strictly monotonically with the upstream gas density. Then we would expect a nonlinear correlation between the hard X-ray and the $\gamma$-ray emissions in a hadronic model (see also Sect. 2.3), since electrons do not play a role in the acceleration energetics and the resulting shock modification, acting de facto as test particles. Therefore we expect the amplitude of the accelerated electron energy distribution to increase with $\rho$, even though its form is entirely determined by the accelerating ion component and is in fact equal to the form of the ion energy distribution. The reason is that only the nuclear component - with its dominant energy density - determines the shock structure, and therefore the form of the accelerated energy spectrum of all ultrarelativistic particles whose energy is high compared to the proton rest energy, including the electrons (as long as their radiation loss can be neglected). In fact, the lack of saturation of the electron acceleration will allow the energy density of nonthermal energetic electrons to increase strictly monotonically with their injection rate, i.e. with gas density, in contrast to the saturation behavior of the nonthermal nuclear particles which limits their injection rate.

Even if the electrons were injected - and thus also accelerated - everywhere with the same intensity, then the amplification of the magnetic field by the ions in the quasi-parallel shock regions will automatically lead to an enhancement of the synchrotron emission in these regions, suggesting a correlation of the synchrotron emission with the hadronic $\gamma$-ray emission. This should be qualitatively similar to the case in the bipolar remnant of SN 1006, even though probably less pronounced in wind bubbles. The bipolarity of SN 1006 has been discussed in detail by Völk et al. (2003). For an experimental discussion of the SN 1006 magnetic field configuration, see Rothenflug et al. (2004).

Summarizing the arguments so far, we might expect a good and perhaps even nonlinear spatial correlation between the synchrotron emission and the production of energetic nuclear particles, as a result of pre-existing correlated density and magnetic field strength inhomogeneities, as well as from field amplification in quasi-parallel shock regions. However, this last condition on nuclear particle injection implies that not all gas density enhancements need to show up as regions of enhanced nuclear energetic particle density and amplified magnetic field strength (Plaga 2008). In other words, not all clouds in the environment of RX J1713.7-3946 need to be regions of enhanced synchrotron and hadronic $\gamma$-ray emission. Some might just be shielded against the injection of ions by an unfavorable magnetic field direction relative to the shock normal.

\subsection{Hadronic $\gamma$-ray emission in the Quasi-Sedov phase}

The previous arguments did not consider the explicit modulation effect of upstream density inhomogeneities in the upstream medium on the strength of nuclear particle acceleration and on the resulting hadronic $\gamma$-ray emission. To address this question we again make use of the assumption that the evolutionary phase of RX J1713.7-3946 is dominated by the swept-up mass from the wind bubble-structured interstellar medium. Then the SNR interior should be subsonic with a roughly uniform total gas and particle pressure $P_{\text {tot }} \propto \rho_{1} V_{\mathrm{s}}^{2}$, as known from the Sedov solution in the case without $\mathrm{CR}$ acceleration. Let us call this phase the Quasi-Sedov phase. If for example, in a situation that deviates from spherical symmetry, the shock reaches regions of different gas density over its surface, then the local shock velocity $V_{\mathrm{s}}$ there will vary with the local upstream gas density $\rho_{1}$ as $V_{\mathrm{s}} \propto \rho_{1}^{-0.5}$. In the Quasi-Sedov phase the nuclear energetic particle pressure $P_{\mathrm{c}}$ is an only slowly varying function of time, locally equal to several 10 percent of $P_{\text {tot }}$ if ion injection is efficient (e.g. Ksenofontov et al. 2005). And this nonthermal energy density is primarily concentrated in the highest-energy particles of the spectrum, i.e. in the VHE range. This implies an essentially uniform VHE hadronic $\gamma$-ray emissivity $q_{\gamma} \propto P_{\mathrm{c}}$ in these regions and therefore a local hadronic $\gamma$-ray production rate $Q_{\gamma}=q_{\gamma} \rho_{\mathrm{d}} \propto \rho_{1}$, since the downstream gas density $\rho_{\mathrm{d}}$ is a fixed fraction of the upstream density $\rho_{1}$ for a strong shock.

Therefore the hadronic $\gamma$-ray emission is approximately proportional to the local gas density. Unless the electron acceleration is anticorrelated with the upstream gas density, the X-ray synchrotron emission will be correlated with the hadronic $\gamma$-ray emission, because $B$ and $\rho$ are almost always spatially correlated.

\subsection{Spatial correlations in the leptonic scenario}

In the highly likely case that the leptonic $\gamma$-ray emission is dominated by the IC emission, the $\gamma$-ray morphology is basically determined by the spatial distribution of the radiating electrons. On the other hand, the synchrotron emission is proportional to the product of the energetic electron density and the local magnetic field energy. In the hypothetical case that the hadronic $\gamma$-ray emission is negligible, the observations would of course require a good spatial correlation between X-ray and IC $\gamma$-ray emission. And, the better this correlation in such a leptonic scenario, the more the magnetic field strength must therefore be spatially uniform.

This is a most unlikely situation for RX J1713.7-3946 given the fact that the gas density appears quite variable around the SNR shell and the remnant appears to be interacting with at least some of these molecular clouds, as CO-observations suggest (Fukui et al. 2003; Moriguchi et al. 2005). This makes a purely leptonic scenario already empirically highly unlikely, independently of any theoretical arguments.

\section{Comparison of the latest $\gamma$-ray spectrum with the previous theoretical model of BV06}

The latest version of the HESS $\gamma$-ray spectrum (Aharonian et al. 2007a) presented in Fig. 1 has not only increased statistical accuracy, but also a flat (hard) spectral shape at the lowest energies of $250 \mathrm{GeV}$, and a smooth extension and fall-off towards the $100 \mathrm{TeV}$ region. For the evaluation of this spectrum the reflectivity changes of the HESS mirrors have been taken into account, leading to a roughly 15 percent increase in flux compared to the 2005 spectrum.

Note that the theoretical $\gamma$-ray spectra, calculated in BV06 and also presented in Fig. 2, correspond to the assumptions that RX J1713.7-3946 was a core collapse supernova SN of type $\mathrm{II} / \mathrm{Ib}$ with a massive progenitor and explosion energy 


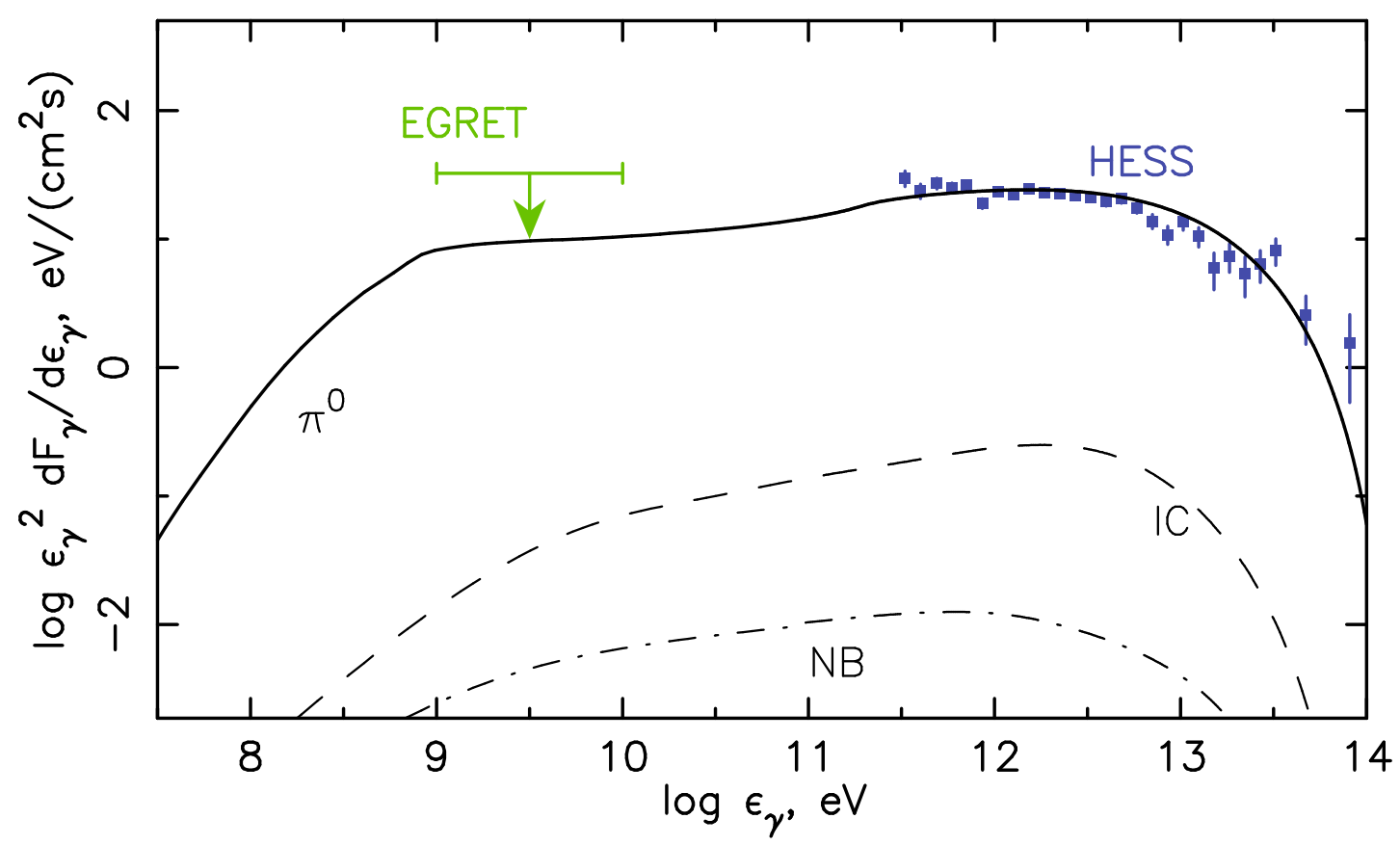

Fig. 1. Spatially integrated, $\gamma$-ray spectral energy distribution of RX J1713.7-3946. The latest HESS $\gamma$-ray data (Aharonian et al. 2007a) (blue color) are shown together with the EGRET upper limit for the RX J1713.7-3946 position (Aharonian et al. 2006) (green color). The solid curve at energies above $10^{7} \mathrm{eV}$ corresponds to $\pi^{0}$-decay $\gamma$-ray emission, whereas the dashed and dash-dotted curves indicate the Inverse Compton (IC) and Nonthermal Bremsstrahlung (NB) emissions, respectively, from the theoretical model of BV06. See also Berezhko \& Völk (2007).

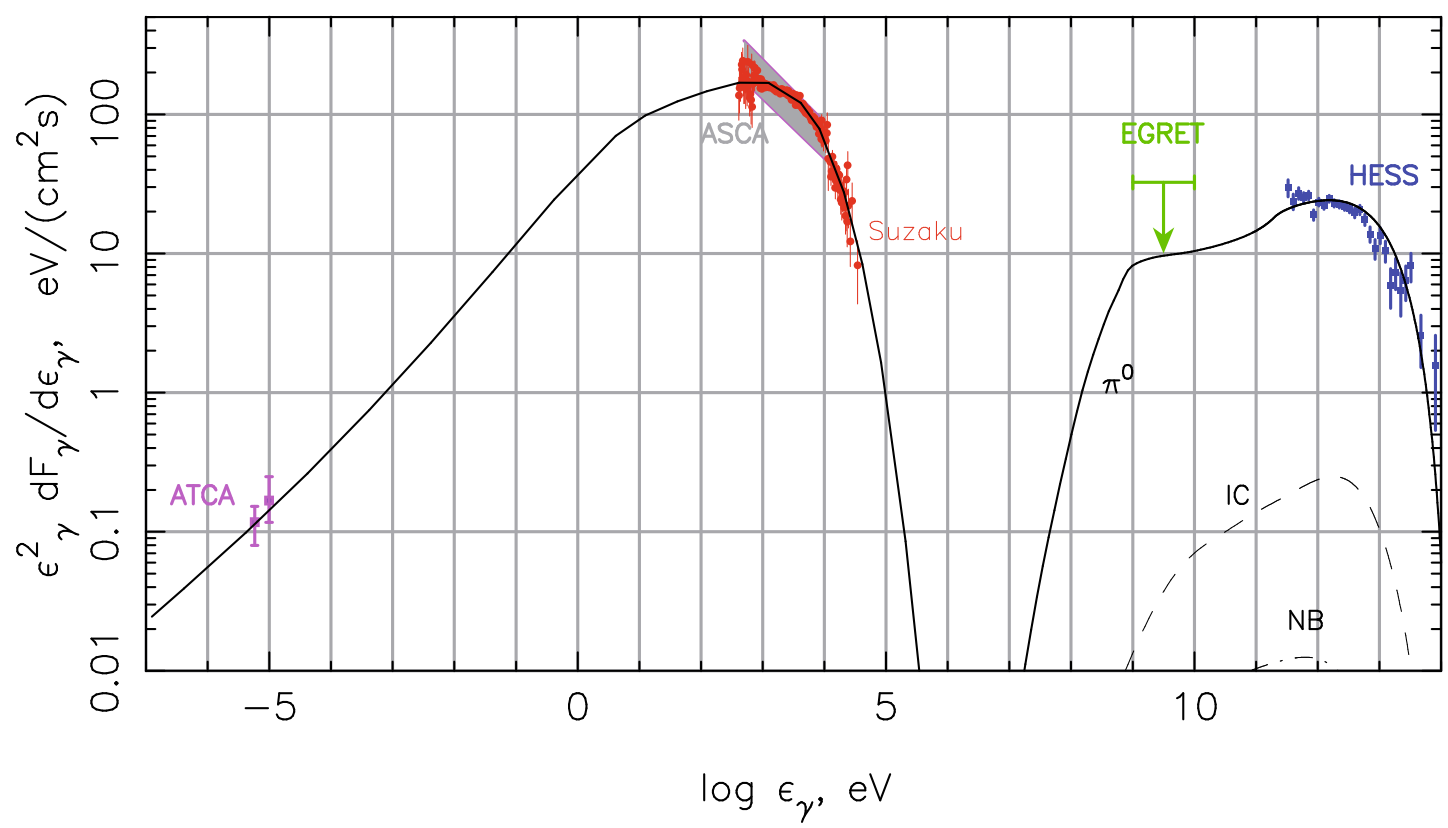

Fig. 2. Spatially integrated, overall nonthermal spectral energy distribution of RX J1713.7-3946. The ATCA radio data (cf. Aharonian et al. 2006) [violet color], ASCA X-ray data (cf. Aharonian et al. 2006), Suzaku X-ray data (Uchiyama et al. 2008) [red color], and 2006 HESS $\gamma$-ray data (Aharonian et al. 2007a) [blue color] are shown. The EGRET upper limit for the RX J1713.7-3946 position (Aharonian et al. 2006) [green color] is shown as well. The solid curve at energies above $10^{7} \mathrm{eV}$ corresponds to $\pi^{0}$-decay $\gamma$-ray emission, whereas the dashed and dash-dotted curves indicate the Inverse Compton (IC) and Nonthermal Bremsstrahlung (NB) emissions, respectively, from the theoretical model of Berezhko \& Völk (2006).

$E_{\text {sn }}=1.8 \times 10^{51} \mathrm{erg}$, that it has an age of $\approx 1600 \mathrm{yr}$ and is located at a distance of $\approx 1 \mathrm{kpc}$. Although this general scenario corresponds to the conclusions of most other authors, significantly larger distances also have been considered in the literature (Slane et al. 1999). For details, see the discussion in BV06.

Since the theoretical model cannot well determine the spectral amplitude, for the reasons given in BV06, the above-mentioned flux increase is not relevant in a comparison of theoretical and observational spectra ${ }^{2}$.

However, the forms of the spectra agree remarkably well. We note that the inferred leptonic IC and Nonthermal

\footnotetext{
2 This is approximately also true if cross-field diffusion of the highestenergy nuclear particles modifies the shock almost everywhere, as discussed in the Introduction.
} 


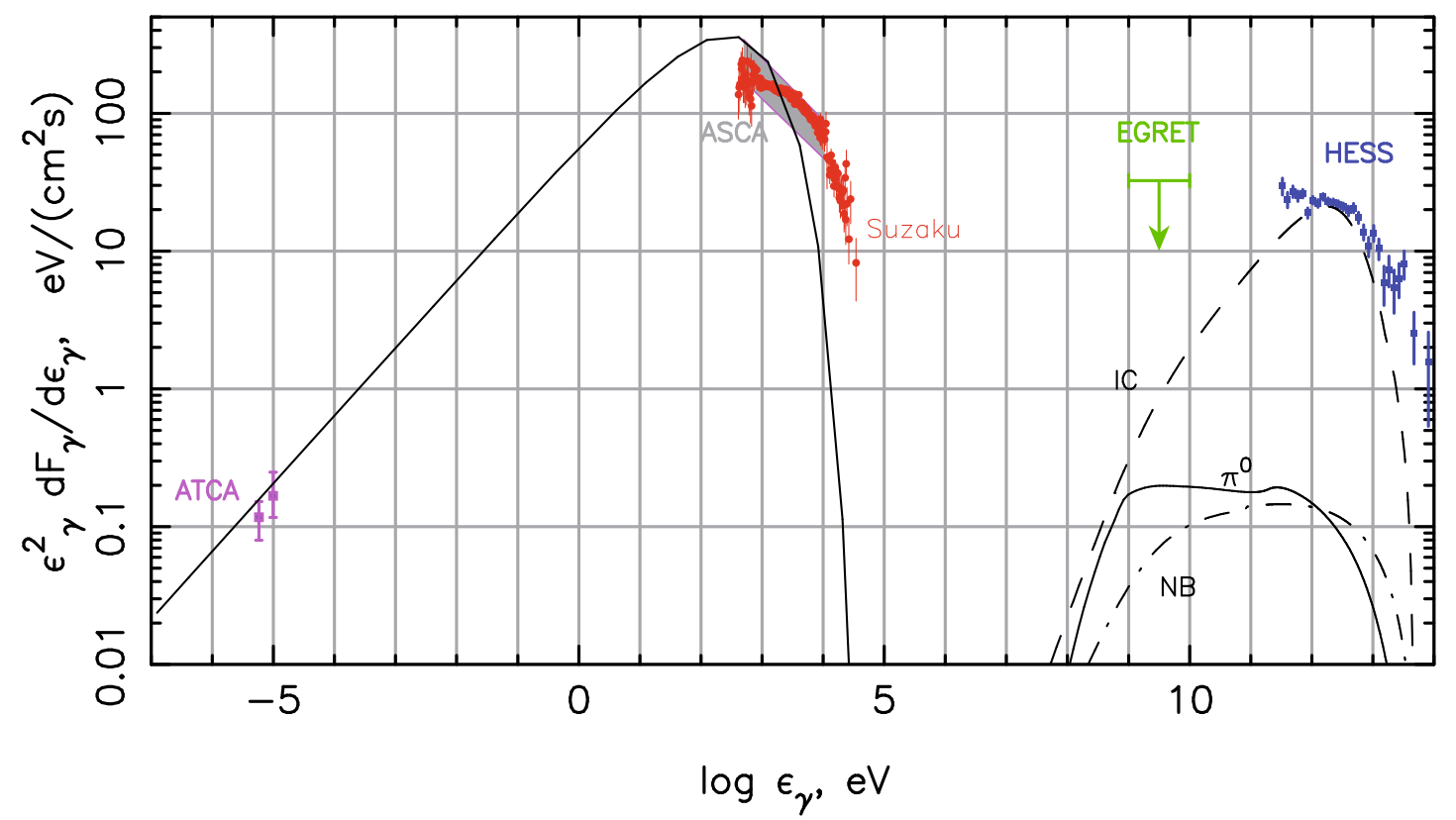

Fig. 3. The same as in Fig. 2, except that a leptonically dominated scenario was assumed (see text).

Bremsstrahlung spectra are depressed by more than an order of magnitude relative to the observed spectrum. They cannot explain the observations, if the magnetic field is indeed amplified to the degree assumed in the theory and supported by the upper limit for the thickness of the synchrotron filaments which one can derive (BV06) from the XMM-observations of this remnant by Hiraga et al. (2005). The resulting lower limit for the magnetic field strength of $65 \mu \mathrm{G}$ has recently been supported by Chandra observation of fast variations of the hard X-ray emission in some spots in the remnant, possibly showing the localized existence of even $\mathrm{mG}$ magnetic field strengths (Uchiyama et al. 2008; see, however also Butt et al. 2008).

\section{Comparison of the latest overall nonthermal spectrum with the BV06 spectrum}

We present in Fig. 2 along with new HESS data the latest hard X-ray spectrum, obtained with the Suzaku instrument (Takahashi et al. 2008), which is given in the form of an energy flux density by Uchiyama et al. (2008), and compare it with the theoretical spectrum of BV06 (see also Zirakashvili \& Aharonian 2007). The Suzaku measurement does not cover the entire SNR, and therefore it needs to be suitably normalized by the requirement of optimum agreement with the ASCA spectrum, cf. Aharonian et al. (2006), over the latter instrument's range between 0.5 and $10 \mathrm{keV}$. The result is shown in Fig. 2. The good agreement basically stems from the fact that the amplified downstream field of $\approx 100 \mu \mathrm{G}$, used to calculate the theoretical spectrum in BV06, already leads to electron synchrotron cooling above an electron momentum of $\approx 500 \mathrm{~m}_{\mathrm{p}} c$, and thus to a hardening of the synchrotron spectrum compared to a spectrum calculated without electron cooling (see Fig. 3 below).

The same observed spectrum can also be compared with a theoretical spectrum (Fig. 3) in which a very low ion injection rate $\left(\eta=10^{-5}\right)$ and a rather low downstream magnetic field strength of $20 \mu \mathrm{G}$ was assumed (see BV06). This corresponds to a dominantly leptonic $\gamma$-ray test particle spectrum without field amplification ${ }^{3}$. The IC-scattered diffuse radiation field is the CMB plus interstellar far infrared and optical radiation fields as given in Berezhko et al. (2003b). This corresponds to typical values used for the Solar neighborhood (e.g. Drury et al. 1994; Gaisser et al. 1998; Porter et al. 2006). We note that for nearby objects at distances $d \sim 1 \mathrm{kpc}$ the CMB contribution dominates in the IC emission (see also Porter et al. 2006).

The electron injection strength was fitted such that an optimum fit to the observations in the radio and X-ray ranges is achieved, cf. Fig. 3. We note that the form of the X-ray spectrum is only very poorly fitted in this leptonic scenario, especially when one uses the recent Suzaku measurements. Also the $\gamma$-ray spectrum has a maximum which is much too sharp in comparison with the observed HESS spectrum. We note that, compared to earlier measurements (Aharonian et al. 2005), the latest version of the HESS $\gamma$-ray spectrum (Aharonian et al. 2007a) deviates more clearly from the IC-type spectrum with a relatively sharp peak at $\epsilon_{\gamma} \sim 1 \mathrm{TeV}$. Note also that our spectrum of the nonthermal emission, that corresponds to the leptonic (or inefficient) scenario, is almost identical to the spectrum presented by Porter et al. (2006), even though they approximate the electron spectrum analytically, whereas we calculate it numerically. Therefore it is also clear that the quality of the fit achieved by Porter et al. (2006) with a leptonic model will be considerably lower if one uses the Suzaku X-ray spectrum and the new HESS $\gamma$-ray spectrum instead of older, less accurate data.

At $\gamma$-ray energies of $1 \mathrm{GeV}$ the spectral energy flux density is a factor of about 30 below the value in the hadronic scenario. It might be possible to construct a more or less plausible form of the diffuse radiation field spectrum to obtain a reasonable fit in the $\mathrm{TeV}$ region. However, it remains very doubtful in our view whether this can avoid the sharp decline towards the GeV energy

\footnotetext{
${ }^{3}$ In fact, the strength of the downstream magnetic field might be even smaller by a factor of two or more, wherever the shock is not locally parallel to the external field. However, adopting such a small field would imply that even the gross amplitude of the maximum of the observed $\gamma$-ray energy flux could not be fitted to the observations.
} 
range indicated in Fig. 3. In any case such a construction cannot improve the unacceptable fit in the hard X-ray range.

\section{Conclusions}

We conclude that a theoretical acceleration model which takes into account magnetic field amplification and a consistent nuclear energetic particle production is consistent with the latest HESS $\gamma$-ray and Suzaku hard X-ray observations. It is also expected to be consistent with the observed good correlation between X-ray synchrotron emission and VHE $\gamma$-ray emission. In the face of existing gas density variations in or near the SNR, a purely leptonic interpretation of the spatial correlation becomes poorer as this correlation becomes closer empirically. The attempt to explain the $\gamma$-ray emission by leptonic processes cannot be made consistent with the observed synchrotron spectrum. The availability of higher-quality hard X-ray and $\gamma$-ray measurements leads to a better consistency with the kinetic nonlinear theory prediction, whereas the consistency of the leptonic model becomes much poorer.

Whether a leptonic model can lead to an acceptable fit of the $\gamma$-ray spectrum through a more detailed evaluation of the local diffuse radiation field is an open question. In our view, even such a modification would encounter enormous difficulties in attempting to fit the $\gamma$-ray spectrum over the additional two orders in $\gamma$-ray energy down from the VHE range to the $\mathrm{GeV}$ range. The leptonic scenario seems also to be inconsistent with the filamentary X-ray morphology which suggests substantial field amplification at least over part of the remnant.

Acknowledgements. The authors would like to thank V. S. Ptuskin, F. A. Aharonian and V. N. Zirakashvili for discussions on the general topic. E.G.B. acknowledges the partial support by the Presidium of RAS (program No. 16) and by the Russian Foundation for Basic Research (grant 07-02-00221) and the hospitality of the Max-Planck-Institut für Kernphysik, where part of this work was carried out.

\section{References}

Aharonian, F. A., Akhperjanian, A., Barrio, J., et al. 2001, A\&A, 370, 112

Aharonian, F. A., Akhperjanian, A., Aye, K.-M., et al. (HESS Collaboration) 2004, Nature, 432, 75

Aharonian, F. A., Akhperjanian, A., Bazer-Bachi, A. R., et al. (HESS Collaboration) 2005, A\&A, 437, L7

Aharonian, F. A., Akhperjanian, A., Bazer-Bachi, A. R., et al. (HESS Collaboration) 2006, A\&A, 449, 223
Aharonian, F. A., Akhperjanian, A., Bazer-Bachi, A. R., et al. (HESS Collaboration) 2007a, A\&A, 464, 235

Aharonian, F. A., Akhperjanian, A., Bazer-Bachi, A. R., et al. (HESS Collaboration) 2007b, ApJ, 661, 236

Albert, J., Aliu, E., Anderhub, H., et al. (MAGIC Collaboration) 2007, A\&A, 474, 937

Bamba, A., Yamazaki, R., Ueno, M., \& Koyama, K. 2003, ApJ, 589, 827

Berezhko, E. G. 2005, Adv. Space Res., 35, 1031

Berezhko, E. G., \& Völk, H. J. 1997, Astropart. Phys., 7, 183

Berezhko, E. G., \& Völk, H. J. 2004, A\&A, 419, L27

Berezhko, E. G., \& Völk, H. J. 2006, A\&A, 451, 981

Berezhko, E. G., \& Völk, H. J. 2007, to be published in Proc. 30th ICRC (Merida) [arXiv:0707.4647]

Berezhko, E. G., Ksenofontov, L. T., \& Völk, H. J. 2002, A\&A, 395, 943

Berezhko, E. G., Ksenofontov, L. T., \& Völk, H. J. 2003a, A\&A, 412, L11

Berezhko, E. G., Pühlhofer, G., \& Völk, H. J. 2003b, A\&A, 400, 971

Berezhko, E. G., Ksenofontov, L. T., \& Völk, H. J. 2006, A\&A, 395, 943

Berezhko, E. G., Pühlhofer, G., \& Völk, H. J. 2008, A\&A, submitted

Butt, Y. M., Porter, T. A., Katz, B., et al. 2008, MNRAS, 386, L20

Drury, L., O’C., Aharonian, F. A., \& Völk, H. J. 1994, A\&A, 287, 959

Enomoto, R., Tanimori, T., Naito, T., et al. 2002, Nature, 416, 823

Enomoto, R., Watanabe, S., Tanimori, T., et al. 2006, ApJ, 652, 1268

Fukui, Y., Moriguchi, Y., Tamura, K., et al. 2003, PASJ, 55, L61

Gaisser, T. K., Protheroe, R. J., \& Stanev, T. 1998, ApJ, 492, 219

Hiraga, J. S., Uchiyama, Y., Takahashi, T., et al. 2005, A\&A, 431, 953

Hoppe, S., \& Lemoine-Goumard, M. for the HESS Collaboration 2007, [arXiv: 0709.4103]

Katagiri, H., Enomoto, R., Ksenofontov, L. T., et al. 2005, ApJ, 619, L163

Katz, B., \& Waxman, E. 2008, JCAP, 01, 1

Koyama, K., Petre, R., Gotthelf, E. V., et al. 1995, Nature, 378, 225

Ksenofontov, L. T., Berezhko, E. G., \& Völk, H. J. 2005, A\&A, 443, 973

Long, K. S., Reynolds, S. P., Raymond, J. C., et al. 2003, ApJ, 586, 1162

Moriguchi, Y., Tamura, T., Tawara, Y., et al. 2005, ApJ, 641, 947

Muraishi, H., Tanimori, T., \& Yanagita, S. 2000, A\&A, 354, L57

Plaga, R. 2008, New Astron., 13, 73

Parizot, E., Marcowith, A., Ballet, J., et al. 2006, A\&A 453, 387

Porter, T. A., Moskalenko, I. V., \& Strong, A. W. 2006, ApJ, 648, L29

Rothenflug, R., Ballet, J., Dubner, G., et al. 2004, A\&A, 425, 121

Slane, P., Gaensler, B. M., Dame, T., et al. 1999, ApJ, 357, SL99

Takahashi, T., Tanaka, T., Uchiyama, Y., et al. 2008, PASJ, 60, S131

Tanaka, T., Uchiyama, Y., Aharonian, F. A., et al. 2008, ApJ, in press [arXiv:0806.1490]

Uchiyama, Y., Aharonian, F. A., Tanaka, T., et al. 2007, Nature, 449, 576

Vink, J., \& Laming, J. M. 2003, ApJ, 548, 758

Völk, H. J. 2008, Adv. Space Res., submitted

Völk, H. J., Berezhko, E. G., Ksenofontov, L. T., \& Rowell, G. P. 2002, A\&A, 396, 649

Völk, H. J., Berezhko, E. G., \& Ksenofontov, L. T. 2003, A\&A, 409, 563

Völk, H. J., Berezhko, E. G., \& Ksenofontov, L. T. 2005, A\&A, 433, 229

Völk, H. J., Berezhko, E. G., \& Ksenofontov, L. T. 2007, Ap\&SS, 309, 389

Völk, H. J., Berezhko, E. G., \& Ksenofontov, L. T. 2008a, A\&A, 483, 529

Völk, H. J., Ksenofontov, L. T., \& Berezhko, E. G., 2008b, A\&A, 490, 515

Zirakashvili, V. N., \& Aharonian, F. A. 2007, A\&A, 465, 695 